\title{
Effect of IL-17 in the development of colon cancer in mice
}

\author{
LIJUAN YANG ${ }^{1,2 *}$, HAO LIU $^{3 *}$, LILI ZHANG ${ }^{4}$, JIE HU $^{5}$, HAIXIA CHEN $^{6}$, \\ LEI WANG $^{7}$, XIAOLIN YIN ${ }^{1,2}$, QUANHAI LI ${ }^{1,8}$ and YIXIN QI ${ }^{9}$
}

\begin{abstract}
${ }^{1}$ Department of Immunology, College of Basic Medical Sciences, Hebei Medical University; ${ }^{2}$ The Key Laboratory of Immune Mechanism and Intervention on Serious Disease in Hebei Province, Shijiazhuang, Hebei 050017; ${ }^{3}$ Department of the Nuclear Medicine, The First Affiliated Hospital of Hebei Medical University, Shijiazhuang, Hebei 050031; ${ }^{4}$ The Second Hospital of Shijiazhuang; ${ }^{5}$ School of Nursing, Hebei Medical University, Shijiazhuang, Hebei 050000; ${ }^{6}$ Department of Electroencephalogram, Xingtai People's Hospital, Xingtai, Hebei 054031; ${ }^{7}$ Department of Pathogenic Biology and Immunology, Cangzhou Medical College, Cangzhou, Hebei 061001; ${ }^{8}$ Cell Therapy Laboratory,

The First Hospital of Hebei Medical University, Shijiazhuang, Hebei 050031; ${ }^{9}$ Department of General Surgery, The Fourth Hospital of Hebei Medical University, Shijiazhuang, Hebei 050011, P.R. China
\end{abstract}

Received December 24, 2015; Accepted June 16, 2016

DOI: $10.3892 / \mathrm{ol} .2016 .5329$

\begin{abstract}
Cytokine therapy is commonly used for tumor immunotherapy. Although early studies focused directly on the tumor, current investigations are more attentive of the tumor microenvironment. Various immune cells and related cytokines in the tumor microenvironment play an important role in the occurrence and development of tumor. Interleukin (IL)-17 is the characteristic cytokine produced by Th17 cells. IL-17 has been associated with various immune responses. The results of previous studies showed that IL-17 can significantly reduce the size of transplanted tumors in tumor-bearing mice, albeit it has no effect on the survival time of mice. By investigating the effect of IL-17 in the number and distribution of lymphocyte infiltration in tumor tissues, the expression of cytokines and transcription factors associated with the subsets of $\mathrm{CD}^{+}{ }^{+} \mathrm{T}$ cells in tumor tissues, the distribution of subsets of spleen lymphocyte in tumor-bearing mice, a preliminary investigation of the possible antitumor mechanism of IL-17 was performed. In conclusion, the antitumor effect of $I L-17$ gene transfection in the colon cancer of mice may be associated with the mechanisms whereby $I L-17$ gene transfection can change the distribution of different subsets of spleen
\end{abstract}

Correspondence to: Dr Quanhai Li, Department of Immunology, College of Basic Medical Sciences, Hebei Medical University, 361 Zhongshan East Road, Shijiazhuang, Hebei 050017, P.R. China E-mail:xpa431@163.com

Dr Yixin Qi, Department of General Surgery, The Fourth Hospital of Hebei Medical University, 12 Jiankang Road, Shijiazhuang, Hebei 050011, P.R. China

E-mail: cnk687@163.com

*Contributed equally

Key words: cytokine therapy, interleukin-17, colon cancer lymphocytes in mice. $I L-17$ gene transfection can increase the number of lymphocyte infiltration in tumor tissues. $I L-17$ gene transfection can promote the high expression of interferon- $\gamma$ in tumor tissue, while reducing the expression of IL-10 and IL-13 factors, thus exerting an antitumor effect.

\section{Introduction}

Colon cancer is a common malignant tumor of the digestive tract, mostly occurring in the boundary of rectum and sigmoid colon $(1,2)$. Clinically, it is more prevalent in males as compared to females. It occurs in the 50-60-year age group, and is the third most prevalent of digestive tract malignant tumors. For many years, the treatment of colon cancer included surgical treatment, chemotherapy and radiotherapy. However, all these methods have some limitations. At present, the biological treatment has become a method of choice secondary to the three conventional treatment methods. It is also one of the most popular research areas. Biological therapy includes many methods such as target therapy, gene therapy and tumor vaccine, of which gene therapy is the most recent research topic (3). Evidence has shown that the occurrence and development of malignant tumor is closely related to gene alteration (3). Gene therapy may change the biological behavior of the tumor cells by correcting or repairing the malignant genes.

Cytokines are a class of small molecule proteins with a variety of biological effects. The members of this family play an important role in the occurrence and development of many tumors, therefore cytokine therapy is commonly used for tumor immunotherapy $(3,4)$. In previous studies the focus was on the tumor itself, whereas increasingly investigations have focused on the tumor microenvironment. The tumor microenvironment is a comprehensive system composed of tumor cells, endothelial cells, fibroblasts, extracellular matrix, and all types of cells associated with immunity and inflammation $(5,6)$. It is the internal environment in which the tumor lies during its occurrence and development (7). The various immune cells 
and related cytokines in the tumor microenvironment play an important role in the occurrence and development of tumor.

Interleukin (IL)-17 is the characteristic cytokine produced by Th17 cells. IL-17 is associated with various immune responses (8). There are varied theories on its mechanism, ranging from the fact that IL-17 can promote tumor metastasis by promoting angiogenesis and enhance the ability of tumor metastasis and invasion in order that IL-17 can inhibit tumor growth by enhancing the immune activity of cytotoxic $\mathrm{T}$ cells. The results of our previous studies showed that IL-17 is capable of significantly reducing the size of transplanted tumors in tumor-bearing mice, but has no influence on their survival time. In the present study, a mouse model was established and successfully transfected with $I L-17$ genes. By investigating the effect of IL-17 in the number and distribution of lymphocyte infiltration in tumor tissues, the expression of cytokines and transcription factors associated with the subsets of $\mathrm{CD}^{+}{ }^{+} \mathrm{T}$ cells in tumor tissues, the distribution of subsets of spleen lymphocytes in tumor-bearing mice, a preliminary discussion on the possible antitumor mechanism of IL-17 was conducted, providing sufficient evidence for targeting tumor therapy in the future and providing a new method for tumor therapy.

\section{Materials and methods}

Cells and tumor tissues. The cells and tumor tissues used in the present study were all obtained from our previous study (9). In brief, $60 \mathrm{BALB} / \mathrm{c} \mathrm{nu} / \mathrm{nu}$ mice (4- to 5-week-old male and female mice; $n=30$ per group) were randomly divided into the control, vector control and experimental groups, $\mathrm{n}=20$ mice per group. C26, C26/pcDNA3.1, and C26/IL-17 cells were injected subcutaneously into the mice of the control group (C26), vector control group (C26/pcDNA3.1) and experimental group (C26/IL-17), respectively. The mice from each group were sacrificed after 5 weeks of the inoculation. The animal experiments were approved by the Animal Experiment and Welfare committee at Hebei Medical University. Tumor tissues were stocked into liquid nitrogen $\left(-196^{\circ} \mathrm{C}\right)$. The spleen lymphocytes and tumor-infiltrating lymphocytes from each group were obtained using density gradient centrifugation and stocked in liquid nitrogen $\left(-196^{\circ} \mathrm{C}\right)$.

Quantitative polymerase chain reaction ( $q P C R)$. Extracted RNA from tumor tissues and spleen cells was obtained from a previous study (9). The expression of cytokines and transcription factors associated with T-cell subset differentiation or effect [i.e., interferon (IFN)- $\gamma$, IL-4, GATA-3 and retinoid-related orphan receptor (ROR)- $\gamma \mathrm{t}$ ] were detected. The primer sequence pairs were designed using Primer5 and NCBI online Primer-BLAST software.

The primer sequence pairs used were: $\beta$-actin sense: 5'-TCACCAGGCATTGCTGACAGG-3' and antisense: 5'-ACTTGCGGTGCACGATGGA-3'; IFN- $\gamma$ sense: 5'-AGC TCATCCGAGTGGTCCAC-3' and antisense: 5'-AAAATT CAAATAGTGCTGGCAGAA-3'; IL-4 sense: 5'-GGGTCT CAACCCCCAGCTA-3' and antisense: 5'-CGAGCTCAC TCTCTGTGGTGTT-3'; IL-12 sense: 5'-TAC TAGAGAGAC TTCTTCCACAACAAGAG-3' and antisense: 5'-TCTGGA CACTCTTCAAGTCCTCATAGA-3'; IL-5 sense: 5'-CCC
ATGAGCACAGTGGTGAA-3' and antisense: 5'-CTCATC GTCTCATTGCTTGTCAA-3'; IL-10 sense: 5'-GCCAAG CCTTATCGGAAATG-3' and antisense: 5'-CTTGATTTC TGGGCCATGCT-3'; IL-13 sense: 5'-CCTGGATTCCCT GACCAACA-3' and antisense: 5'-GGGCCTTGCGGTTAC AGA-3'; IL-17 sense: 5'-AAGCTCAGCGTGTCCAAACA-3' and antisense: 5'-TGCGCCAAGGGAGTTAAAGA-3'; transforming growth factor- $\beta$ sense: 5'-TGACGTCACTGGAGT TGTACGG-3' and antisense: 5'-GGTTCATGTCATGGA TGGTGC-3'; IL-23 sense: 5'-AATAATGTGCCCCGTATC CA-3' and antisense: 5'-CTGGAGGAGTTGGCTGAGTC-3'; GATA-3 sense: 5'-CCTACCGGGTTCGGATGTAAGT-3' and antisense: 5'-AGTTCGCGCAGGATGTCC-3'; ROR- $\gamma$ t sense: 5'-TCCAGACAGCCACTGCATTC-3' and antisense: 5'-GTG CGCTGCCGTAGAAGGT-3'; Foxp3 sense: 5'-CTGCTCCTC CTATTCCCGTAAC-3' and antisense: 5'-AGCTAGAGGCTT TGCCTTCG-3'; T-bet sense: 5'-CAACAACCCCTTTGC CAAAG-3' and antisense: 5'-TCCCCCAAGCAGTTG ACAGT-3'.

Western blot analysis. Extracted total proteins from tumor tissues were obtained from a previous study (9). Protein concentrations were determined by Nanodrop ND-1000 (Gene Co., Ltd., Hong Kong, China). Once protein concentrations were determined, $50 \mu \mathrm{l}$ of the protein specimens were denatured in $50 \mu \mathrm{l}$ of $2 \mathrm{X}$ sample buffer $[125 \mathrm{mmol} / 1$ Tris-HCl, $\mathrm{pH} 6.8$, $20 \%$ glycerol, $10 \% \beta$-mercaptoethanol, $0.02 \%$ bromophenol blue, and $4 \%$ sodium dodecyl sulphate (SDS)]. Briefly, $30 \mu \mathrm{g}$ of denatured protein were separated using SDS-polyacrylamide gel electrophoresis and transferred to a polyvinylidene fluoride membrane, which was blocked in 5\% milk proteins that were suspended in tris-buffered saline Tween-20 (TBST) for $2 \mathrm{~h}$ at room temperature. The membrane was rinsed three times with TBST, followed by incubation with the primary antibody rabbit anti-mouse IL-17 (polyclonal, bs-1183R) at a 1:500 dilution; rabbit anti-mouse IL-13 (polyclonal, bs-0560R) (both from Beijing Biosynthesis Biotechnology, Beijing, China) at a 1:500 dilution; rabbit anti-mouse IFN- $\gamma$ (polyclonal, 15365-1-AP) at a 1:500 dilution; rabbit anti-mouse IL-10 (polyclonal, 20850-1-AP) (both from Proteintech, USA) at a 1:500 dilution, rabbit anti-mouse IL-12 (polyclonal, bs-0767R, Beijing Biosynthesis Biotechnology) at a 1:500 dilution; rabbit anti-mouse T-bet (polyclonal, 13700-1-AP) at a 1:200 dilution; rabbit anti-mouse ROR- $\gamma$ t (polyclonal, 13205-1-AP) at a 1:200 dilution; and rabbit anti-mouse Foxp-3 (polyclonal, 22228-1-AP) (all from Proteintech) at a 1:500 dilution overnight at $4^{\circ} \mathrm{C}$. The membrane was then washed and treated with anti-rabbit secondary antibodies (polyclonal, BS13278; Bioworld, China) that were conjugated with horseradish peroxidase at a 1:5,000 dilution. The immunoreactive proteins were visualized with a chemiluminescence detection kit (PerkinElmer, Inc., Waltham, MA, USA), and the expression of glyceraldehyde-3-phosphate dehydrogenase protein was used as the loading control across all the samples analyzed using western blotting.

Hematoxylin and eosin $(H \& E)$ staining. The difference of the number of lymphocyte infiltrations in tumor tissues of mice inoculated with C26/IL-17, C26 and C26/pcDNA3.1 cells was investigated. In a previous study (9), at 35 days after tumor 

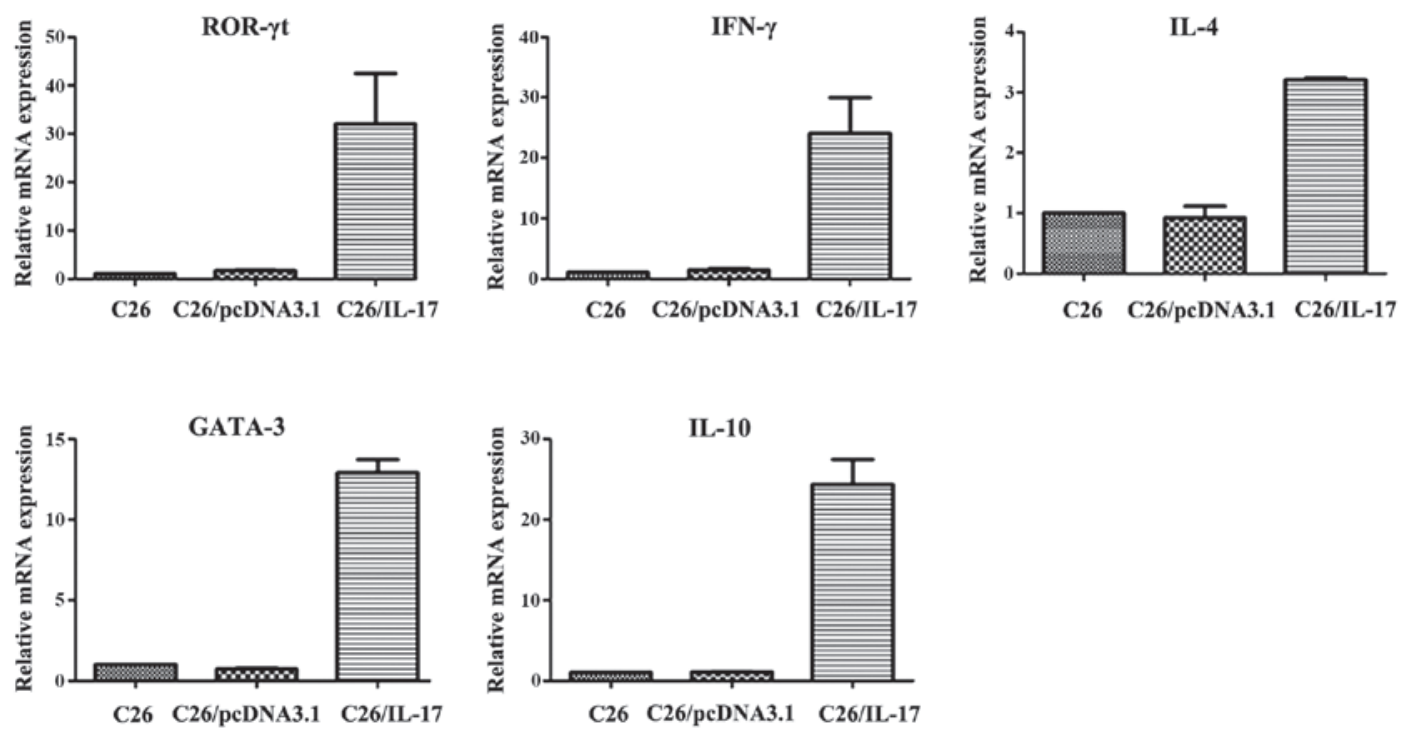

Figure 1. High expression of cytokines and transcription factors in splenocytes from the mice inoculated with C26/interleukin (IL)-17 cells by quantitative polymerase chain reaction (qPCR) $(\mathrm{P}<0.05)$. Inoculated C26 cells, C26/pcDNA3.1 cells, C26/IL-17 cells into the back of mice, and executed the mice 35 days later. Applied qPCR to detect the expression of cytokines and transcription factors in the spleen lymphocyte of mice. Results showed that compared with the mice inoculated with C26 cells and C26/pcDNA3.1cells, the spleen lymphocyte of mice inoculated with C26/IL-17 cells had higher expression of retinoid-related orphan receptor (ROR)- $\gamma$ t, interferon (IFN)- $\gamma$, IL-4, GATA-3 and IL-10 mRNA. The expression of mRNA of ROR- $\gamma$ t, IFN- $\gamma$, IL-4, GATA-3 and IL-10 in spleen lymphocyte of mice inoculated with C26 cells and C26/pcDNA3.1 cells had no significant difference (P>0.05).

cell inoculation in mice, the tumor tissues of mice in each group, were fixed in $10 \%$ formalin $(\mathrm{pH}$ 6.8-7.2) for $\geq 24 \mathrm{~h}$. Paraffin-embedded blocks were prepared, and $4-\mu \mathrm{m}$ sections were cut. Sections $(4 \mu \mathrm{m})$ were dewaxed using xylene and washed with alcohol and water, followed by hematoxylin staining for $5 \mathrm{~min}$. The sections were washed with tap water, differentiated with hydrochloric acid for $30 \mathrm{sec}$, soaked in tap water for $15 \mathrm{~min}$, and stained with eosin for $2 \mathrm{~min}$. The sections were then dehydrated, cleared and mounted. The difference in the number of lymphocyte infiltrations in tumor tissues was measured. Images of the sections were captured using a Positive electric microscope (U-MCZ, Olympus, Tokyo, Japan) at a high-power field magnification of x400. The number of lymphocytes under a single field of vision were counted. Subsequently, five fields were selected for each section and the average value was calculated.

Statistical analysis. Statistical analysis was performed using SPSS 21.0 software (IBM SPSS, Armonk, NY, USA). Normal distribution test (Kolmogorov-Smirnov test) was used to assess the enumeration data and the one-way ANOVA test was used to evaluate the data with equal variance. The Kruskal-Wallis test was used for data without normal distribution or equal variance. Measurement data were presented as mean \pm standard deviation. $\mathrm{P}<0.05$ was considered to indicate a statistically significant difference.

\section{Results}

Effect of IL-17 gene transfection on the distribution of subsets of splenic cells in tumor-bearing mice. C26, C26/pcDNA3.1, and C26/IL-17 cells were inoculated into the back of mice. The mice were sacrificed 35 days thereafter. qPCR was applied to detect the expression of the characteristic cytokine (IL-17) and transcription factor (ROR- $\gamma \mathrm{t}$ ) of Th17 cells, characteristic
Table I. Expression of cytokines and transcription factors in spleen lymphocytes from different mice (mean \pm standard deviation).

\begin{tabular}{lccc}
\hline & \multicolumn{3}{c}{ Group } \\
\cline { 2 - 4 } Factors & C26 & C26/pcDNA3.1 & \multicolumn{1}{c}{ C26/IL-17 } \\
\hline ROR- $\gamma$ t & $1 \pm 0$ & $1.690 \pm 0.096$ & $32.076 \pm 10.399^{\mathrm{a}}$ \\
IL-17 & $1 \pm 0$ & $1.066 \pm 0.193$ & $0.560 \pm 0.248^{\mathrm{a}}$ \\
IFN- $\gamma$ & $1 \pm 0$ & $1.439 \pm 0.185$ & $24.043 \pm 5.921^{\mathrm{a}}$ \\
T-bet & $1 \pm 0$ & $1.066 \pm 0.119$ & $0.297 \pm 0.018^{\mathrm{a}}$ \\
IL-4 & $1 \pm 0$ & $0.923 \pm 0.193$ & $3.207 \pm 0.028^{\mathrm{a}}$ \\
GATA-3 & $1 \pm 0$ & $0.705 \pm 0.079$ & $12.921 \pm 0.793^{\mathrm{a}}$ \\
IL-10 & $1 \pm 0$ & $1.080 \pm 0.047$ & $24.393 \pm 3.077^{\mathrm{a}}$ \\
Foxp-3 & $1 \pm 0$ & $0.676 \pm 0.120$ & $0.558 \pm 0.143^{\mathrm{a}}$ \\
\hline
\end{tabular}

${ }^{\mathrm{a}} \mathrm{C} 26 / \mathrm{IL}-17$ compared to the C26/pcDNA3.1 and C26 groups, $\mathrm{P}<0.05$. IL, interleukin; INF, interferon; ROR- $\gamma \mathrm{t}$, retinoid-related orphan receptor- $\gamma \mathrm{t}$.

cytokine (IFN- $\gamma$ ) and transcription factor (T-bet) of Th1 cells, characteristic cytokine (IL-4) and transcription factor (GATA-3) of Th2 cells, and the characteristic cytokine (IL-10) and transcription factor (Foxp-3) of Treg cells in the spleen lymphocyte of mice.

The results showed that compared with the mice inoculated with C26 and C26/pcDNA3.1 cells, the spleen lymphocytes of mice inoculated with C26/IL-17 cells had a higher expression of ROR- $\gamma$ t, IFN- $\gamma$, IL-4, GATA-3 and IL-10 mRNA (Fig. 1, Table I) but a lower expression of IL-17, T-bet and Foxp-3 mRNA $(\mathrm{P}<0.05)$ (Fig. 2). Additionally, the expression of mRNA of IL-17, ROR- $\gamma$ t, IFN- $\gamma$, T-bet, IL-4, 

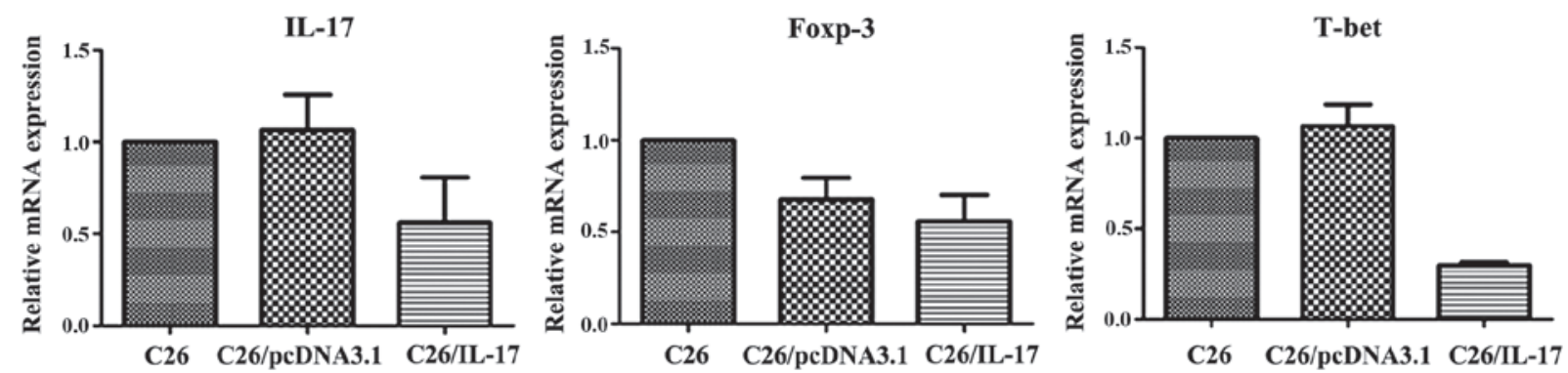

Figure 2. A lower expression of cytokines and transcription factors in splenocytes from the mice inoculated with C26/interleukin (IL)-17 cells by quantitative polymerase chain reaction (qPCR) $(\mathrm{P}<0.05)$. C26, C26/pcDNA3.1, and C26/IL-17 cells were treated as described in Materials and methods. The results show that compared with the mice inoculated with C26 and C26/pcDNA3.1 cells, the spleen lymphocyte of mice inoculated with C26/IL-17 cells had a lower expression of IL-17, T-bet and Foxp-3 mRNA. The mRNA expression of IL-17, T-bet and Foxp-3 in spleen lymphocyte of mice inoculated with C26 and C26/pcDNA3.1 cells had no significant difference $(\mathrm{P}>0.05)$.
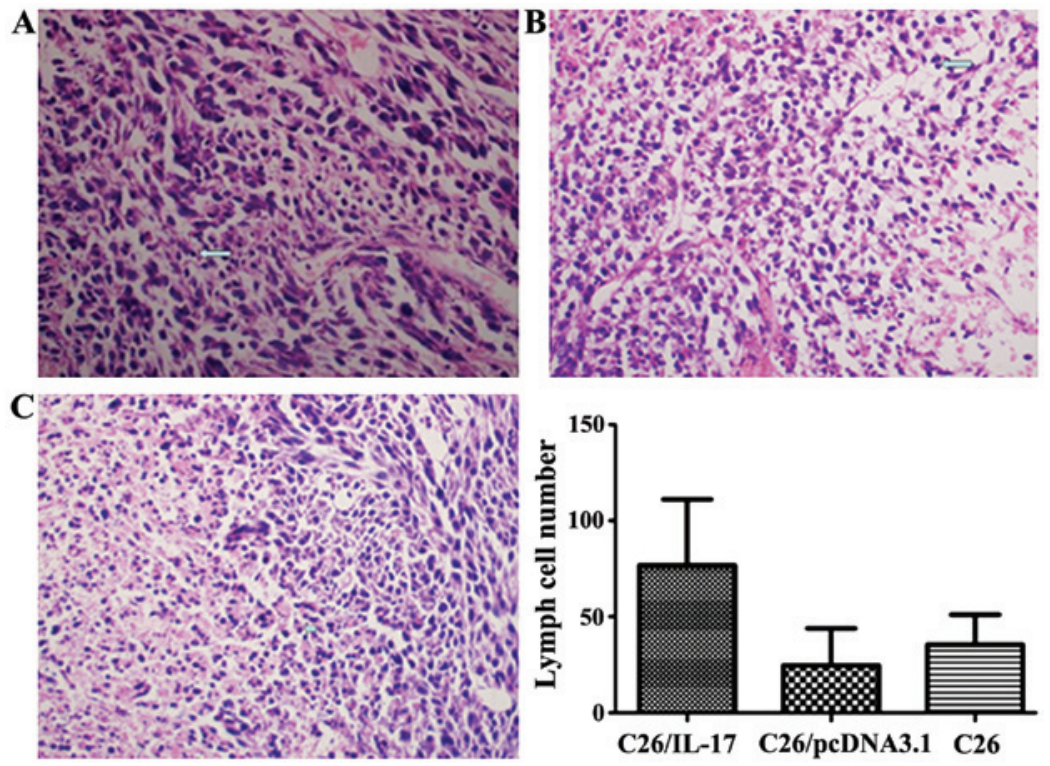

Figure 3. The number of lymphocytes infiltrated in different mice tumor tissue (P<0.05). (A) C26, (B) C26/pcDNA3.1, (C) C26/interleukin (IL)-17 were analyzed using a microscope at a magnification of $\mathrm{x} 400 . \mathrm{C} 26, \mathrm{C} 26 / \mathrm{pcDNA} 3.1$, and C26/IL-17 cells were inoculated into the back of mice, and the mice were sacrificed 35 days later. The tumor tissue of mice in each group were removed, formalin-fixed and prepared in paraffin, sectioned and hematoxylin and eosin (H\&E) staining was performed. The difference of the number of lymphocyte infiltrations in tumor tissues of mice inoculated with C26/IL-17, C26 and C26/pcDNA3.1 cells was identified. H\&E results show that the number of lymphocyte infiltrations in tumor tissues of mice inoculated with C26/IL-17 cells was significantly more than the other two groups $(\mathrm{P}<0.05)$. The number of lymphocyte infiltration in tumor tissues of mice inoculated with $\mathrm{C} 26$ and C26/pcDNA3.1 cells had no significant difference $(\mathrm{P}>0.05)$.

GATA-3, IL-10 and Foxp-3 in the spleen lymphocyte of mice inoculated with C26 cells and C26/pcDNA3.1 cells had no significant difference $(\mathrm{P}>0.05)$.

Effect of IL-17 gene transfection on lymphocyte infiltration in tumor tissues. The H\&E results showed that the number of lymphocyte infiltration in tumor tissues of mice inoculated with C26/IL-17 cells was significantly more than the other two groups $(\mathrm{P}<0.05)$ (Fig. 3, Table II). The number of lymphocyte infiltration in tumor tissues of mice inoculated with C26 and C26/pcDNA3.1 cells had no significant difference $(\mathrm{P}>0.05)$.

Effect of IL-17 gene transfection on the expression of cytokines in the tumor tissues of mice. $\mathrm{C} 26, \mathrm{C} 26 / \mathrm{pcDNA} 3.1$, and C26/IL-17 cells were inoculated into the back of mice, and the mice were sacrificed 35 days later. The expression of

Table II. The number of lymphocytes infiltrated in tumor tissue of different mice.

Group

$\mathrm{C} 26$

C26/pcDNA3.1

C26/IL-17/male

${ }^{a}$ Compared C26/IL-17 to C26/pcDNA3.1 and C26 group, $\mathrm{P}<0.05$. IL, interleukin.

cytokines IL-17 and IL-23 was detected in Th17 cells, IFN- $\gamma$ and IL-12 in Th1 cells, IL-4 and IL-13 in Th2 cells, and IL-10 in Treg cells. 

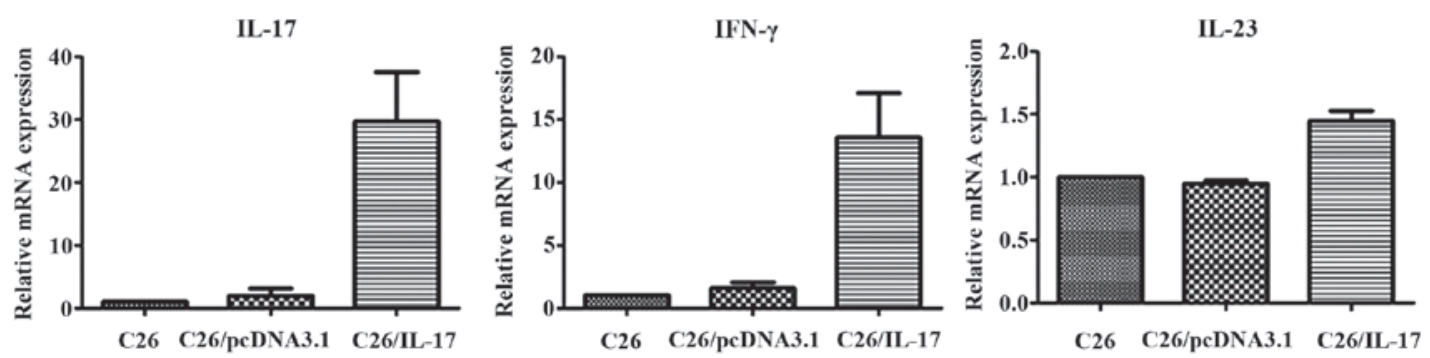

Figure 4. High expression of cytokines in the $\mathrm{C} 26 /$ interleukin (IL)-17 mice tumor tissue by quantitative polymerase chain reaction (qPCR) (P<0.05). C26, C26/pcDNA3.1, and C26/IL-17 cells were inoculated into the back of mice, and the mice were sacrificed 35 days later. The expression of cytokines of tumor tissues was detected. The qPCR results show that, compared with the mice inoculated with C26 and C26/pcDNA3.1 cells, the tumor tissues of mice inoculated with C26/IL-17 cells had a higher expression of IL-17, interferon (IFN)- $\gamma$ and IL-23 mRNA(P<0.05). mRNA expression of the above cytokines in the tumor tissues of mice inoculated with $\mathrm{C} 26$ and $\mathrm{C} 26 / \mathrm{pcDNA} 3.1$ cells had no significant difference $(\mathrm{P}>0.05)$.
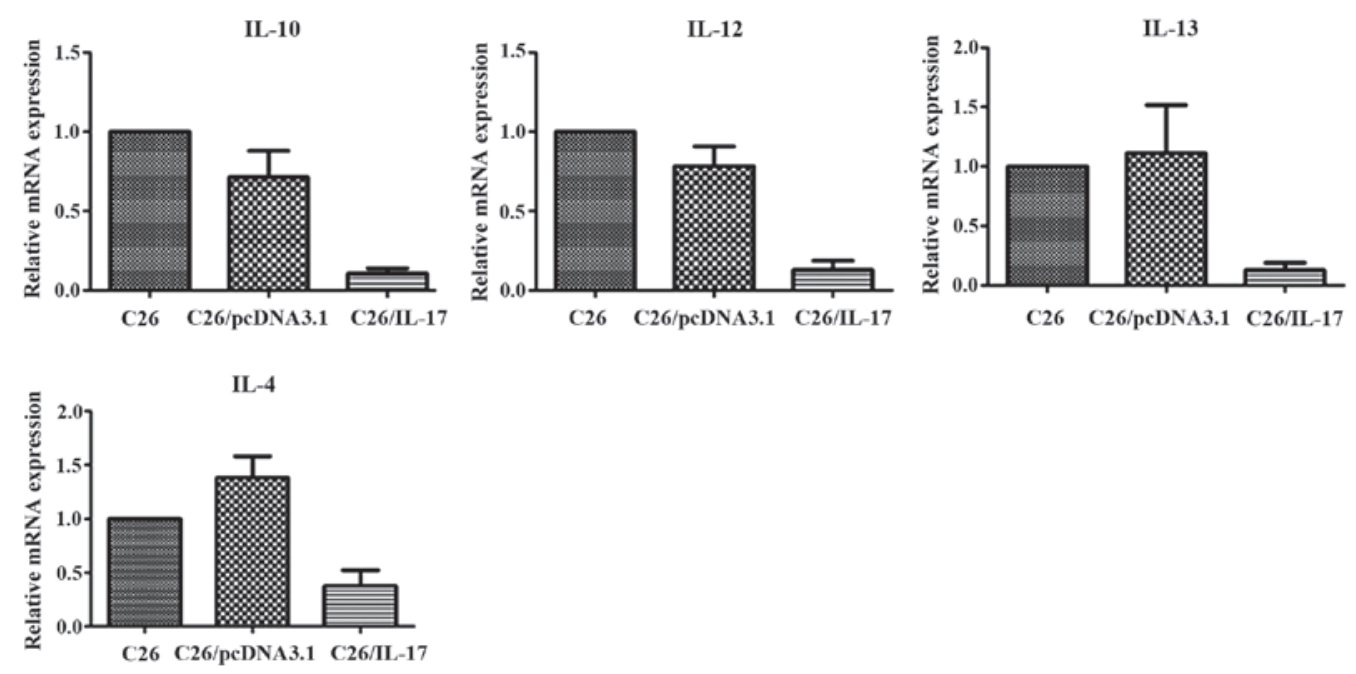

Figure 5. Lower expression of cytokines in the $\mathrm{C} 26 /$ interleukin (IL)-17 mice tumor tissue using quantitative polymerase chain reaction (qPCR) ( $<<0.05$ ). C26, C26/pcDNA3.1, and C26/IL-17 cells were inoculated into the back of mice, and the mice were sacrificed 35 days later. The expression of cytokines of tumor tissues was detected. The qPCR results show that, compared with the mice inoculated with C26 and C26/pcDNA3.1 cells, the tumor tissues of mice inoculated with C26/IL-17 cells had a lower expression of IL-4, IL-10, IL-12 and IL-13 mRNA $(\mathrm{P}<0.05)$. mRNA expression of the above cytokines in the tumor tissues of mice inoculated with $\mathrm{C} 26$ and $\mathrm{C} 26 / \mathrm{pcDNA} 3.1$ cells had no significant difference $(\mathrm{P}>0.05)$.

The qPCR results showed that, compared with the mice inoculated with C26 and C26/pcDNA3.1 cells, the tumor tissues of mice inoculated with C26/IL-17 cells had a higher expression of IL-17, IFN- $\gamma$ and IL-23 mRNA, and differences were statistically significant $(\mathrm{P}<0.05)$ (Fig. 4, Table III), whereas a lower expression of IL-4, IL-10, IL-12 and IL-13 mRNA was identified $(\mathrm{P}<0.05)$ (Fig. 5). The mRNA expression of the above cytokines in the tumor tissues of mice inoculated with C26 cells and C26/pcDNA3.1 cells had no significant difference $(\mathrm{P}>0.05)$.

The western blot results revealed that, compared with the mice inoculated with $\mathrm{C} 26$ and C26/pcDNA3.1 cells, the tumor tissues of mice inoculated with C26/IL-17 cells had a higher expression of IL-17, IFN- $\gamma$ protein (Fig. 6) but a lower expression of IL-10, IL-12 and IL-13 protein. The expression of IL-17, IFN- $\gamma$, IL-10, IL-12 and IL-13 proteins in the tumor tissues of mice inoculated with C26 and C26/pcDNA3.1 cells had no significant difference $(\mathrm{P}>0.05)$.

Expression of characteristic transcription factors of Th1, Th2, Th17, and Treg cells in the tumor tissues of tumor-bearing mice. C26, C26/pcDNA3.1, and C26/IL-17 cells were inoculated into
Table III. Expression of cytokines and transcription factors in tumor tissue from different mice (mean \pm standard deviation).

\begin{tabular}{lccc}
\hline & \multicolumn{3}{c}{ Group } \\
\cline { 2 - 4 } Factors & C26 & C26/pcDNA3.1 & C26/IL-17 \\
\hline ROR- $\gamma$ t & $1 \pm 0$ & $1.485 \pm 0.412$ & $34.221 \pm 12.598^{\mathrm{a}}$ \\
IL-17 & $1 \pm 0$ & $3.833 \pm 1.197$ & $42.408 \pm 7.863^{\mathrm{a}}$ \\
IFN- $\gamma$ & $1 \pm 0$ & $1.572 \pm 0.449$ & $13.573 \pm 3.529^{\mathrm{a}}$ \\
IL-23 & $1 \pm 0$ & $0.945 \pm 0.028$ & $1.448 \pm 0.080^{\mathrm{a}}$ \\
IL-4 & $1 \pm 0$ & $1.491 \pm 1.414$ & $0.360 \pm 0.116^{\mathrm{a}}$ \\
GATA-3 & $1 \pm 0$ & $1.232 \pm 0.269$ & $0.500 \pm 0.086^{\mathrm{a}}$ \\
IL-10 & $1 \pm 0$ & $0.713 \pm 0.166$ & $0.107 \pm 0.032^{\mathrm{a}}$ \\
Foxp-3 & $1 \pm 0$ & $1.162 \pm 0.137$ & $0.301 \pm 0.169^{\mathrm{a}}$ \\
IL-12 & $1 \pm 0$ & $0.780 \pm 0.120$ & $0.130 \pm 0.060^{\mathrm{a}}$ \\
IL-13 & $1 \pm 0$ & $1.112 \pm 0.406$ & $0.128 \pm 0.061^{\mathrm{a}}$ \\
\hline
\end{tabular}

${ }^{\mathrm{a}} \mathrm{C} 26 / \mathrm{IL}-17$ compared to the C26/pcDNA3.1 and C26 groups, $\mathrm{P}<0.05$. IL, interleukin; INF, interferon; ROR- $\gamma \mathrm{t}$, retinoid-related orphan receptor- $\gamma \mathrm{t}$. 


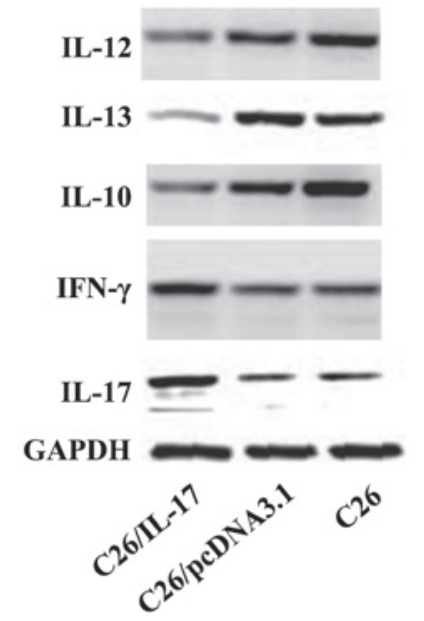

Figure 6. The protein levels of interleukin (IL)-17, interferon (IFN)- $\gamma$, IL-10, IL-12, IL-13 in different mouse tumor tissue by western blotting. C26, C26/pcDNA3.1, and C26/IL-17 cells were inoculated into the back of mice, and the mice were sacrificed 35 days later. The expression of cytokines of tumor tissues was detected. Western blotting results show that, compared with the mice inoculated with C26 and C26/pcDNA3.1 cells, the tumor tissues of mice inoculated with C26/IL-17 cells had a higher expression of IL-17, and IFN- $\gamma$ protein, but a lower expression of IL-10, IL-12 and IL-13 protein. The expression of IL-17, IFN- $\gamma$, IL-10, IL-12 and IL-13 proteins in the tumor tissues of mice inoculated with C26 and C26/pcDNA3.1 cells had no significant difference $(\mathrm{P}>0.05)$.

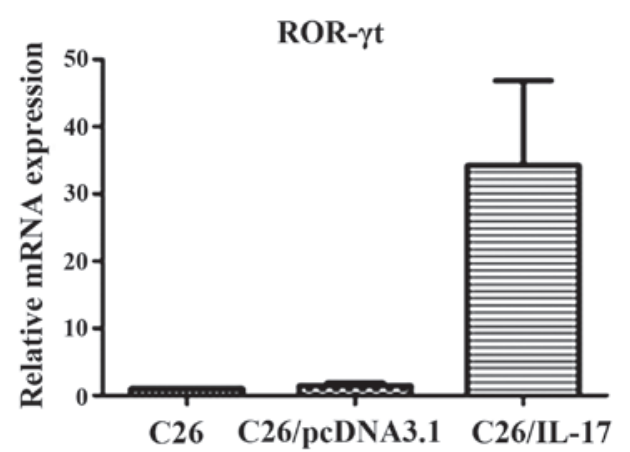

Figure 7. High expression of transcription factors in the C26/interleukin (IL)-17 mouse tumor tissue by quantitative polymerase chain reaction (qPCR) $(\mathrm{P}<0.05)$. C26, C26/pcDNA3.1, and C26/IL-17 cells were inoculated into the back of mice, and the mice were sacrificed 35 days later. qPCR results show that compared with the mice inoculated with C26 and C26/pcDNA3.1 cells, the tumor tissues of mice inoculated with C26/IL-17 cells had a higher expression of retinoid-related orphan receptor $(\mathrm{ROR})-\gamma \mathrm{t}$ mRNA $(\mathrm{P}<0.05)$. mRNA expression of ROR- $\gamma \mathrm{t}$ in the tumor tissues of mice inoculated with C26 and C26/pcDNA3.1 cells had no significant difference ( $P>0.05)$.

the back of mice, and the mice were sacrificed 35 days later. The expression of ROR- $\gamma \mathrm{t}$ associated with Th17 cells, T-bet with Th1 cells, GATA-3 with Th2 cells, and Foxp-3 with Treg cells was detected.

The qPCR results showed that compared with the mice inoculated with C26 and C26/pcDNA3.1 cells, the tumor tissues of mice inoculated with C26/IL-17 cells had a higher expression of ROR- $\gamma \mathrm{t}$ mRNA $(\mathrm{P}<0.05)($ Fig. 7$)$ but a lower expression of GATA-3 and Foxp-3 mRNA, and differences were statistically significant $(\mathrm{P}<0.05)$ (Fig. 8). The mRNA expression of the above transcription factors in the tumor tissues of mice inoculated with $\mathrm{C} 26$ and C26/pcDNA3.1 cells had no significant difference $(\mathrm{P}>0.05)$.
The western blotting results showed that, compared with the mice inoculated with $\mathrm{C} 26$ and C26/pcDNA3.1 cells, the tumor tissues of mice inoculated with C26/IL-17 cells had a higher expression of ROR- $\gamma$ t protein (Fig. 9). The expression of ROR- $\gamma$ t protein in the tumor tissues of mice inoculated with $\mathrm{C} 26$ and C26/pcDNA3.1 cells had no significant difference $(\mathrm{P}>0.05)$.

\section{Discussion}

IL-17 is a cytokine with multiple biological effects. It may be produced by NK T cells, $\mathrm{CD} 8^{+} \mathrm{T}$ cells, $\gamma \delta \mathrm{T}$ cells, dendritic cells, macrophages and other cells (9), but is mainly produced by Th17 cells (10). Th17 is a newly identified T-helper cell subset. Its appearance has challenged the traditional classification of $\mathrm{CD} 4^{+} \mathrm{T}$-cell subsets. Previous findings have shown that Th17 cell was characterized by secreting cytokine IL-17 (9). Clinical data have shown that IL-17 was involved in the occurrence of many types of autoimmune diseases and inflammation, and also closely associated with the occurrence and development of tumor. However, its role in tumor is controversial. IL-17 is known to promote tumor by promoting angiogenesis, inhibiting tumor cell apoptosis and promoting tumor metastasis and invasion (11-14). By contrast, IL-17 is considered to inhibit tumor by enhancing the activity of NK cells, promoting the activation and production of CTL cells and inhibiting the infiltration of tumor cells (15-24).

Radosavljevic et al (25) demonstrated that IL-17 is an important indicator of the development of colon cancer. In a previous study, we successfully established mouse models of colon cancer and identified that $I L-17$ gene transfection may significantly reduce the tumor size of tumor-bearing mice. This finding may be associated with its antitumor effect. In the current study, we investigated the antitumor mechanism of IL-17 in mice with colon cancer.

It is known that the various subsets of $\mathrm{CD} 4^{+} \mathrm{T}$ cells are of great significance in inhibiting tumors and Th1, Th2, Th17 and Treg cells are most closely associated with tumors. Therefore, in the current study four types of $\mathrm{CD} 4{ }^{+} \mathrm{T}$ cells were used. The results of qPCR showed that compared with the mice inoculated with $\mathrm{C} 26$ and C26/pcDNA3.1 cells, the tumor tissues of mice inoculated with C26/IL-17 cells had an increased expression of ROR- $\gamma \mathrm{t}$ but a decreased expression of IL-17 $(\mathrm{P}<0.05)$, indicating that IL-17 increases the number of ROR- $\gamma \mathrm{t}^{+}$cells in the spleen of the tumor-bearing mice but reduces the number of Th17 cells. In addition, compared with the mice inoculated with $\mathrm{C} 26$ and $\mathrm{C} 26 / \mathrm{pcDNA} 3.1$ cells, the tumor tissues of mice inoculated with C26/IL-17 cells had an elevated expression of IFN- $\gamma$ but a reduced expression of T-bet $(\mathrm{P}<0.05)$, indicating that IL-17 can increase the number of IFN- $\gamma^{+}$cells in spleen of tumor-bearing mice, but reduce the number of TH1 cells. Compared with the mice inoculated with C26 and C26/pcDNA3.1 cells, the tumor tissues of mice inoculated with C26/IL-17 cells had a higher expression of IL-4 and GATA-3 $(\mathrm{P}<0.05)$, indicating that IL-17 can increase the number of Th2 cells in tumor-bearing mice. In addition, compared with the mice inoculated with $\mathrm{C} 26$ and C26/pcDNA3.1 cells, the tumor tissues of mice inoculated with C26/IL-17 cells had a higher expression of IL-10 but a lower expression of Foxp-3 $(\mathrm{P}<0.05)$, indicating that 

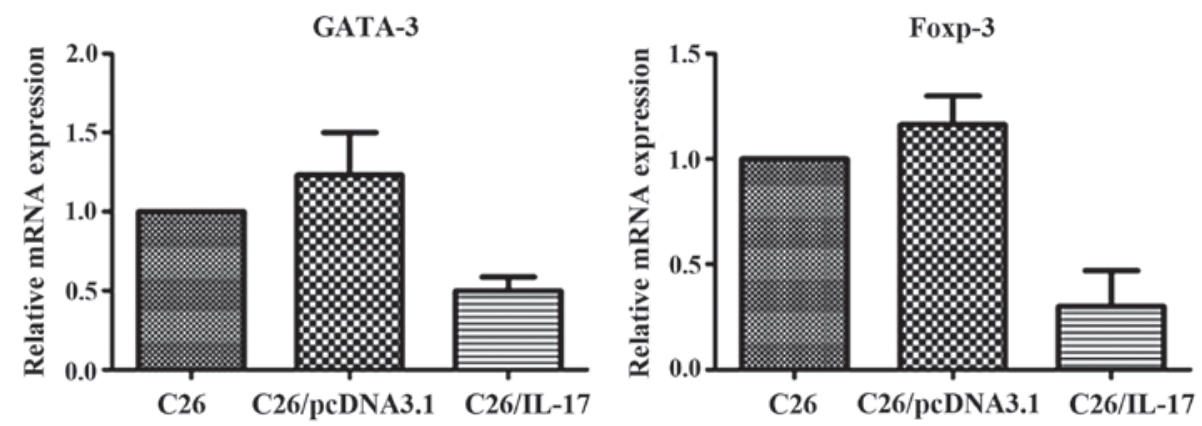

Figure 8. Lower expression of transcription factors in the C26/interleukin (IL)-17 mouse tumor tissue by quantitative polymerase chain reaction (qPCR) $(\mathrm{P}<0.05)$. C26, C26/pcDNA3.1, and C26/IL-17 were inoculated into the back of mice, and the mice were sacrificed 35 days later. The qPCR results show that compared with the mice inoculated with C26 and C26/pcDNA3.1 cells, the tumor tissues of mice inoculated with C26/IL-17 cells had a lower expression of GATA-3 and Foxp-3 mRNA, and differences were statistically significant $(\mathrm{P}<0.05)$. mRNA expression of the above transcription factors in the tumor tissues of mice were inoculated with C26 and C26/pcDNA3.1 cells had no significant difference ( $\mathrm{P}>0.05)$.

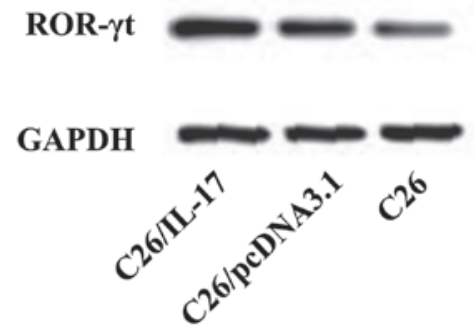

Figure 9. The protein levels of retinoid-related orphan receptor (ROR)- $\gamma \mathrm{t}$ in different mouse tumor tissue by western blotting. C26, C26/pcDNA3.1, and C26/interleukin (IL)-17 cells were inoculated into the back of mice, and the mice were sacrificed 35 days later. Western blotting results show that, compared with the mice inoculated with $\mathrm{C} 26$ and C26/pcDNA3.1 cells, the tumor tissues of mice inoculated with $\mathrm{C} 26 / \mathrm{IL}-17$ cells had a higher expression of ROR- $\gamma t$ protein $(\mathrm{P}<0.05)$. The expression of ROR- $\gamma \mathrm{t}$ protein in tumor tissues of mice inoculated with C26 and C26/pcDNA3.1 cells had no significant difference $(\mathrm{P}>0.05)$.

IL-17 can decrease the number of Foxp- $3^{+}$cells in spleen of tumor-bearing mice. Thus, the increased IL-10 may be derived from Th2 cells. mRNA expression in the various cytokines and transcription factors in the splenocyte of tumor-bearing mice inoculated with C26 and C26/pcDNA3.1 cells had no significant difference $(\mathrm{P}>0.05)$. The above results indicated that $I L-17$ gene may exert an antitumor effect by affecting the distribution of the subsets of spleen cells. The specific phenotype and characteristics of ROR- $\gamma \mathrm{t}^{+}$cells with a low expression of IL-17 and the IFN- $\gamma^{+}$cells with a low expression of T-bet remain to be investigated.

Tumor microenvironment is an essential internal environment in the development of tumor. It is a comprehensive system composed of tumor cells, endothelial cells, fibroblasts, extracellular matrix, and cells associated with immunity and inflammation $(5,6)$. In this complex system, cytokines and various immune cells interact with each other and cooperate to regulate the occurrence, development and invasion and metastasis of tumor. In this study, we conducted an in-depth investigation on the cytokines and Th1, Th2, Th17 and Treg cells in tumor tissues of tumor-bearing mice. Firstly, we applied H\&E staining to count the number of infiltrating lymphocytes in tumor tissues. The results showed that $I L-17$ gene transfection is capable of increasing the number of lymphocytes in the tissues of colon cancer, and the data were statistically significant $(\mathrm{P}<0.05)$, indicating that $I L-17$ gene may exert an antitumor effect by increasing the infiltration of lymphocytes. We also detected the transcription factors associated with Th subsets in the colon cancer tissues and identified that mRNA and proteins of ROR- $\gamma \mathrm{t}$ in tumor tissues of mice inoculated with C26/IL-17 cells, were significantly more than the mice inoculated with C26 and C26/pcDNA3.1 cells, and differences were statistically significant $(\mathrm{P}<0.05)$, indicating that $I L-17$ gene transfection can increase the number of ROR- $\gamma \mathrm{t}^{+}$cells in tumor tissues. Compared with the mice inoculated with C26 and C26/pcDNA3.1 cells, the mice inoculated with C26/IL-17 cells had a lower expression of Foxp-3 and GATA-3 mRNA $(\mathrm{P}<0.05)$, indicating that $I L-17$ gene transfection can reduce the number of Th2 and Treg cells in tumor tissues of mice. The above results indicated that IL-17 could, not only increase the number of TIL, but also regulate the distribution of Th subsets. The antitumor effect of IL-17 may be associated with its effect in increasing the number of TIL and ROR- $\gamma \mathrm{t}^{+}$cells, and reducing the number of Th2 and Treg cells.

We also observed cytokines associated with the differentiation and function of Th subsets in the colon cancer tissues of mice. The results show that compared with the mice inoculated with C26 and C26/pcDNA3.1 cells, the mRNA and proteins of IL-17 in mice inoculated with C26/IL-17 cells were increased, and differences were statistically significant $(\mathrm{P}<0.05)$, indicating that we successfully established $\mathrm{C} 26$ cells that steadily transfected $I L-17$ gene, and the successfully established tumor cells effectively expressed IL-17 mRNA and protein. A number of studies have shown that IFN- $\gamma$ significantly enhanced immunity. It has been previously shown that the growth ability of melanoma and bladder cancer cells in mice with $I F N-\gamma$ gene defect was greatly intensified (14). The current results showed that $I L-17$ gene transfection can significantly increase the mRNA and protein of IFN- $\gamma$ in colon cancer tissues of mice, and the data were statistically significant $(\mathrm{P}<0.05)$. The largely increased IFN- $\gamma$ may be from the $\mathrm{T}$ or NK cells of $\mathrm{CD}^{+}$. Treg cells have been generally recognized as a cell with immunosuppressive action and its inhibitory effect was closely associated with the secretion of IL-10. The results of the present study have shown that $I L-17$ gene transfection can lower the mRNA and protein expression of IL-10 in tumor tissue of tumor-bearing mice, which may be 
associated with the plasmid. However, the $I L-17$ gene further reduced the number of Treg cells. A previous study identified that the role of IL-13 in tumor was the same as IL-10 $(26,27)$. Both were able to promote the tumor growth by inhibiting immunity. The results of our study showed that $I L-17$ gene transfection can reduce the expression of IL-13 in colon cancer tissues. From the above analysis, we suggest that $I L-17$ gene exerts an antitumor effect by increasing the expression of IFN- $\gamma$ and reducing the expression of IL-10 and IL-13.

In conclusion, the antitumor effect of $I L-17$ gene transfection in the colon cancer of mice may be associated with the following mechanisms: i) $I L-17$ gene transfection can change the distribution of different subsets of spleen lymphocytes in mice; ii) $I L-17$ gene transfection can increase the number of lymphocyte infiltration in tumor tissues; and iii) $I L-17$ gene transfection can promote the high expression of IFN- $\gamma$ in tumor tissue, while reducing the expression of IL-10 and IL-13 factors, thus exerting an antitumor effect. Overall, we predict that IL-17 directly or indirectly induces the polarization of tumor tissue infiltration lymphocytes and exerts an anti-tumor effect, which requires further investigation.

\section{Acknowledgements}

The present study was supported by grant nos. 10396106D, 13397703D, 14967719D, C2010000474 and 2011101.

\section{References}

1. Yang TM: Clinical pathological features and survival rate of 116 cases of colorectal cancer. Chin J Med Guide 16: 602-603, 2014

2. Zhao S and Xue B: Clinical and pathological features of rectal cancer in young patients. Mod Oncol 21: 1296-1298, 2013.

3. Zhang C, Wang Q-T, Liu H, Zhang ZZ and Huang WL: Advancement and prospects of tumor gene therapy. Chin J Cancer 30: 182-188, 2011.

4. Hou L-N, He X-H and Zhang Z-X: The present situation and outlook of the treatment of tumor with cytokine gene. J Clin Exp Med 7: 145-147, 2008.

5. Sung SY, Hsieh CL, Wu D, Chung LW and Johnstone PA: Tumor microenvironment promotes cancer progression, metastasis, and therapeutic resistance. Curr Probl Cancer 31: 36-100, 2007.

6. Nelson D, Fisher S and Robinson B: The 'Trojan Horse' approach to tumor immunotherapy: Targeting the tumor microenvironment. J Immunol Res 2014: 789069, 2014.

7. Egeblad M, Nakasone ES and Werb Z: Tumors as organs: Complex tissues that interface with the entire organism. Dev Cell 18: 884-901, 2010.

8. Jie L, Zhou X-H, Qian S, et al: The function of IL-17 family and their roles in related diseases. Curr Immunol 34: 262-265, 2014.

9. Korn T, Bettelli E, Oukka M and Kuchroo VK: IL-17 and Th17 Cells. Annu Rev Immunol 27: 485-517, 2009.

10. Fossiez F, Djossou O, Chomarat P, Flores-Romo L, Ait-Yahia S, Maat C, Pin JJ, Garrone P, Garcia E, Saeland S, et al: $\mathrm{T}$ cell interleukin-17 induces stromal cells to produce proinflammatory and hematopoietic cytokines. J Exp Med 183: 2593-2603, 1996.
11. Wakita D, Sumida K, Iwakura Y, Nishikawa H, Ohkuri T, Chamoto K, Kitamura $\mathrm{H}$ and Nishimura T: Tumor-infiltrating IL-17-producing gammadelta $\mathrm{T}$ cells support the progression of tumor by promoting angiogenesis. Eur J Immunol 40: 1927-1937, 2010.

12. He D, Li H, Yusuf N, Elmets CA, Li J, Mountz JD and Xu H: IL-17 promotes tumor development through the induction of tumor promoting microenvironments at tumor sites and myeloid-derived suppressor cells. J Immunol 184: 2281-2288, 2010.

13. Dhodapkar KM, Barbuto S, Matthews P, Kukreja A, Mazumder A, Vesole D, Jagannath S and Dhodapkar MV: Dendritic cells mediate the induction of polyfunctional human IL17-producing cells (Th17-1 cells) enriched in the bone marrow of patients with myeloma. Blood 112: 2878-2885, 2008.

14. Wang L, Yi T, Kortylewski M, Pardoll DM, Zeng D and Yu H: IL-17 can promote tumor growth through an IL-6-Stat3 signaling pathway. J Exp Med 206: 1457-1464, 2009.

15. Hirahara N, Nio Y, Sasaki S, Minari Y, Takamura M, Iguchi C, Dong M, Yamasawa $\mathrm{K}$ and Tamura K: Inoculation of human interleukin-17 gene-transfected Meth-A fibrosarcoma cells induces $\mathrm{T}$ cell-dependent tumor-specific immunity in mice. Oncology 61: 79-89, 2001.

16. Benchetrit F, Ciree A, Vives V, Warnier G, Gey A, Sautès-Fridman C, Fossiez F, Haicheur N, Fridman WH and Tartour E: Interleukin-17 inhibits tumor cell growth by means of a T-cell-dependent mechanism. Blood 99: 2114-2121, 2002.

17. Hirahara N, Nio Y, Sasaki S, Takamura M, Iguchi C, Dong M, Yamasawa K, Itakura M and Tamura K: Reduced invasiveness and metastasis of Chinese hamster ovary cells transfected with human interleukin-17 gene. Anticancer Res 20 (5A): 3137-3142, 2000.

18. Martin-Orozco N, Muranski P, Chung Y, Yang XO, Yamazaki T, Lu S, Hwu P, Restifo NP, Overwijk WW and Dong C: T helper 17 cells promote cytotoxic $\mathrm{T}$ cell activation in tumor immunity. Immunity 31: 787-798, 2009.

19. Yang ZZ, Novak AJ, Ziesmer SC, Witzig TE and Ansell SM: Malignant $\mathrm{B}$ cells skew the balance of regulatory $\mathrm{T}$ cells and TH17 cells in B-cell non-Hodgkin's lymphoma. Cancer Res 69: 5522-5530, 2009

20. Horlock C, Stott B, Dyson PJ, Morishita M, Coombes RC, Savage $\mathrm{P}$ and Stebbing J: The effects of trastuzumab on the CD4+CD25+FoxP3+ and CD4+IL17A+ T-cell axis in patients with breast cancer. Br J Cancer 100: 1061-1067, 2009.

21. Muranski P, Boni A, Antony PA, Cassard L, Irvine KR, Kaiser A, Paulos CM, Palmer DC, Touloukian CE, Ptak K, et al: Tumor-specific Th17-polarized cells eradicate large established melanoma. Blood 112: 362-373, 2008.

22. Sfanos KS, Bruno TC, Maris CH, Xu L, Thoburn CJ, DeMarzo AM, Meeker AK, Isaacs WB and Drake CG: Phenotypic analysis of prostate-infiltrating lymphocytes reveals TH17 and Treg skewing. Clin Cancer Res 14: 3254-3261, 2008.

23. Dougan $M$ and Dranoff G: Inciting inflammation: The RAGE about tumor promotion. J Exp Med 205: 267-270, 2008.

24. Kryczek I, Wei S, Szeliga W, Vatan L and Zou W: Endogenous IL-17 contributes to reduced tumor growth and metastasis. Blood 114: 357-359, 2009.

25. Radosavljevic G, Ljujic B, Jovanovic I, Srzentic Z, Pavlovic S, Zdravkovic N, Milovanovic M, Bankovic D, Knezevic M, Acimovic LJ, et al: Interleukin-17 may be a valuable serum tumor marker in patients with colorectal carcinoma. Neoplasma 57: 135-144, 2010.

26. Zhang J-J and Zheng W-M: The relationship of IL-13 and its receptor to malignant tumor. Foreign Med Sci Oncol Sect 32: 736-739, 2005.

27. Terabe M, Park JM and Berzofsky JA: Role of IL-13 in regulation of anti-tumor immunity and tumor growth. Cancer Immunol Immunother 53: 79-85, 2004. 